\title{
(6) OPEN ACCESS Are there three main subgroups within the
patellofemoral pain population? A detailed
characterisation study of 127 patients to help
develop targeted intervention (TIPPS)
}

\author{
James Selfe, ${ }^{1}$ Jessie Janssen, ${ }^{1}$ Michael Callaghan, ${ }^{2}$ Erik Witvrouw, ${ }^{3}$ Chris Sutton, ${ }^{1}$ \\ Jim Richards, ${ }^{1}$ Maria Stokes, ${ }^{4}$ Denis Martin, ${ }^{5}$ John Dixon, ${ }^{5}$ Russell Hogarth, ${ }^{1}$ \\ Vasilios Baltzopoulos, ${ }^{6}$ Elizabeth Ritchie, ${ }^{7}$ Nigel Arden, ${ }^{8}$ Paola Dey ${ }^{1}$
}

\begin{abstract}
${ }^{1}$ University of Central Lancashire, Preston, Lancashire, UK ${ }^{2}$ Institute for Inflammation and Repair, Centre for Musculoskeletal Research, University of Manchester, Manchester, UK ${ }^{3}$ Aspetar, Orthopaedic and Sports Medicine Hospital, Doha, Qatar

${ }^{4}$ Faculty of Health Sciences, University of Southampton, Southampton, UK

${ }^{5}$ Teesside University,

Middlesbrough, UK

${ }^{6}$ Brunel University London, Uxbridge, UK

${ }^{7}$ Department of Physiotherapy, Harrogate \& District NHS Foundation Trust, Harrogate District Hospital, Harrogate, North Yorkshire, UK ${ }^{8}$ University of Oxford, Nuffield Orthopaedic Centre, Oxford, UK
\end{abstract}

Correspondence to Professor James Selfe, School of Sport Tourism and the Outdoors, University of Central Lancashire, Preston PR1 2HE, UK; JSelfe1@uclan.ac.uk

Accepted 16 November 2015 Published Online First 1 February 2016

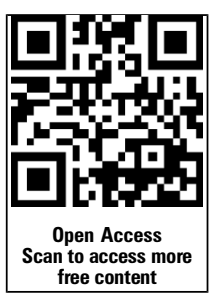

CrossMark

To cite: Selfe J, Janssen J, Callaghan $\mathrm{M}$, et al. $\mathrm{Br} \mathrm{J}$ Sports Med 2016;50: 873-880.

\section{ABSTRACT}

Background Current multimodal approaches for the management of non-specific patellofemoral pain are not optimal, however, targeted intervention for subgroups could improve patient outcomes. This study explores whether subgrouping of non-specific patellofemoral pain patients, using a series of low cost simple clinical tests, is possible.

Method The exclusivity and clinical importance of potential subgroups was assessed by applying à priori test thresholds (1 SD) from seven clinical tests in a sample of adult patients with non-specific patellofemoral pain. Hierarchical clustering and latent profile analysis, were used to gain additional insights into subgroups using data from the same clinical tests.

Results 130 participants were recruited, 127 had complete data: 84 (66\%) female, mean age 26 years (SD 5.7) and mean body mass index 25.4 (SD 5.83), median (IQR) time between onset of pain and assessment was $24(7-60)$ months. Potential subgroups defined by the à priori test thresholds were not mutually exclusive and patients frequently fell into multiple subgroups. Using hierarchical clustering and latent profile analysis three subgroups were identified using 6 of the 7 clinical tests. These subgroups were given the following nomenclature: (1) 'strong', (2) 'weak and tighter' and (3) 'weak and pronated foot'.

Conclusions We conclude that three subgroups of patellofemoral patients may exist based on the results of six clinical tests which are feasible to perform in routine clinical practice. Further research is needed to validate these findings in other data sets and, if supported by external validation, to see if targeted interventions for these subgroups improve patient outcomes.

\section{BACKGROUND}

Non-specific patellofemoral pain (PFP) is a musculoskeletal disorder of the knee joint that causes significant pain and dysfunction around the patella leading to limitations in physical activities. ${ }^{1}$ The condition is not self-limiting, $90 \%$ of patients with PFP still have symptoms 4 years after diagnosis, ${ }^{2}{ }^{3}$ and only $6 \%$ are symptom free at 16 years follow-up. ${ }^{4}$

PFP may be a risk factor for developing patellofemoral osteoarthritis (OA). ${ }^{5} 6$ PFP has recently emerged as the third highest ranked topic out of 185 in the Chartered Society of Physiotherapy
(UK) Musculoskeletal Research Priority Project. ${ }^{7}$ Expert consensus statements published following three International Patellofemoral Pain Research Retreats (IPFPRR) propose biomechanical risk factors for developing PFP described by anatomical location relative to the knee. These factors are: Proximal-upper femur, hip and trunk; Local-in and around the patella and the patellofemoral joint; Distal-lower leg foot and ankle. ${ }^{8-10}$ These risk factors may guide in developing clinical subgroups.

Subgrouping approaches have proved fruitful to optimise management in other musculoskeletal conditions such as low back pain, in which psychosocial characteristics have also been incorporated into subgroup criteria. ${ }^{11} 12$ Previous authors have investigated subgroups within the PFP population using specialised high cost equipment not routinely seen in clinic for example, radiographic examination and scintigraphy, ${ }^{13}$ dynamic MRI ${ }^{14}{ }^{15}$ and six camera three-dimensional motion analysis systems. ${ }^{16}$ The translation of these results into routine practice in physiotherapy clinics is therefore likely to be limited. With the exception of Dierks et $a l^{16}$ the focus of these studies has tended to be on local biomechanical factors, rather than adopting an holistic approach that also incorporates proximal and distal factors.

Recently Selhorst et $a l^{17}$ reported on a pilot study of 21 paediatric patients with PFP, mean age 14 years, where they defined a new PFP classification algorithm that contains four subgroups; elevated fear avoidance, decreased muscle flexibility, functional malalignment, decreased muscle strength. Unfortunately they provided no details are as to how the four groups were derived. Keays et $a l^{18}$ also described four clinical PFP subgroups; hypermobility, hypomobility, faulty movement pattern, osteoarthritis. Interestingly they had a very wide age range in their sample from 13 to 82 years with only eight patients in the 20-40 year age range and each participant was required to have four X-rays of the knee. With the exception of gender ${ }^{19}$ the main focus of most previous studies of PFP has been on biomechanical rather than nonbiomechanical factors. ${ }^{20}$ Selhorst et al ${ }^{17}$ highlight the necessity of addressing psychosocial factors in PFP and there is some evidence to suggest a relationship exists between patients with PFP and 
activity levels, ${ }^{8}$ weight ${ }^{6}$ and pain mechanisms, ${ }^{21}$ these factors may be of relevance to subgrouping approaches in the management of patients suffering from PFP.

The present study is part of a larger programme of work exploring whether targeted management of PFP subgroups can optimise patient outcome. Previously, seven clinical assessment tests have been proposed that may be useful in identifying clinical subgroups. ${ }^{22}$ Further detail on these clinical assessment tests and à priori test thresholds can be found in the protocol for this study. $^{22}$

In the present paper, we aimed to explore whether subgrouping of patellofemoral pain patients, using a series of low cost simple clinical tests, was possible. Four objectives were identified:

1. To determine the relative frequency with which the patients fell into each of the potential subgroups defined by the à priori test thresholds;

2. To assess whether the potential subgroups defined by the à priori test thresholds were mutually exclusive or whether, and how frequently, patients fell into two or more subgroups;

3. To ascertain whether other approaches such as hierarchical clustering and latent profile analysis, offered additional insights into subgrouping of patients with PFP using data from the same clinical assessment tests;

4. To report differences in patient-related characteristics (demographic, clinical and psychosocial) across subgroups.

\section{METHODS}

\section{Design of the clinical study}

A cross-sectional observational study design was used. Participants attended an assessment clinic on one occasion prior to physiotherapy treatment, at which a physiotherapist undertook the seven clinical assessment tests (table 1). In addition, an assessment of demographic (eg, age, gender and anthropometry), clinical (eg, skin temperature index, time since onset), and psychosocial characteristics (eg, physical activity, function, quality of life and pain levels) was completed (table 2). No formal power calculation was undertaken given the exploratory nature of the study but a target sample of 150 was considered sufficient to estimate the proportion of participants who fell into different subgroups and for multiple group membership, with adequate precision. ${ }^{22}$

\section{The setting and type of participants}

Four National Health Service (NHS) physiotherapy clinics, serving the general population, participated in this study; one in primary care, three in hospital settings. Between May 2012 and November 2013, patients aged between 18 and 40 years diagnosed with non-specific unilateral or bilateral PFP were approached to participate in this study (figure 1). Eligibility criteria were based on criteria used in two previous studies 2324 where patients with specific pathologies such as ligamentous instability or patella subluxation were excluded, these are fully detailed in the protocol for this study. ${ }^{22}$ Patients were included in this study if the duration of their pain was at least 3 months and they self-reported anterior or retropatellar pain on at least two of the following activities: prolonged sitting, ascending or descending stairs, squatting, running, kneeling and hopping/ jumping. Two of the following on clinical examination were also required: pain during resisted isometric quadriceps contraction, pain on palpation of the posterior facets of the patella, pain during squatting. When eligible patients agreed to participate, written informed consent was obtained.

\section{Clinical assessment}

There were seven clinical assessment tests (1) passive prone knee flexion (rectus femoris length), (2) passive knee extension in supine (hamstrings length), (3) calf flexibility standing method (gastrocnemius length) measured using inclinometry, (4) Hip abductor strength, (5) quadriceps strength measured using handheld dynamometry, (6) Total patellar mobility (medial plus lateral glide) measured using the patellar glide test and (7) foot pronation assessed by the Foot Posture Index (FPI). In the presence of bilateral PFP pain, assessment was undertaken on the most affected leg, as nominated by the patient. To ensure standardisation across the study centres, all therapists attended an initial, and three refresher, training days and were provided with a manual outlining the assessment procedures. Each site was visited by members of the research team (JS and JJ) on at least three occasions to monitor fidelity with assessment procedures.

\section{Statistical methods}

For each measure of muscle flexibility, the mean of three assessments, reported in degrees from the baseline position, was taken as the test score. For both measures of muscle strength, the maximum moment measured in Newton-metre $(\mathrm{Nm})$ from three trials was taken as the test score. This was also normalised to body mass that is Newton-metre per kilogram $(\mathrm{Nm} / \mathrm{kg})$. For patellar mobility the total medial/lateral displacement in millimetres of the distal pole was taken as the test score. For the seven clinical assessment tests, and patient demographic, clinical, psychosocial and functional characteristics, summaries are presented using mean (SD), median (IQR) or frequency (\%), as appropriate.

In this paper we report the findings of an exploratory analysis of the membership of predetermined subgroups using two approaches. First the data were explored using à priori test thresholds, based on 1 SD from published norms (table 3). The lower limb biarticular muscle tightness subgroup was defined by lack of flexibility in two of the three clinical assessment tests. For quadriceps and hip abductor muscle weakness subgroups, the test

Table 1 Mean (SD) for the 7 clinical tests for 127 participants

\begin{tabular}{|c|c|c|}
\hline \multirow[t]{9}{*}{ Clinical assessment tests } & Rectus Femoris Length test ${ }^{\circ}$ & $129.4(20.05)$ \\
\hline & Hamstring Length test ${ }^{\circ}$ & $151.4(14.77)$ \\
\hline & Gastrocnemius Length test ${ }^{\circ}$ & $27.8(10.75)$ \\
\hline & Maximum Quadriceps Strength Nm & $73.7(41.13)$ \\
\hline & Maximum Quadriceps Strength normalised to body mass Nm/kg & $1.0(0.51)$ \\
\hline & Maximum Hip Abductor Strength Nm & $72.8(38.85)$ \\
\hline & Maximum Hip Abductor Strength normalised to body weight Nm/kg & $1.0(0.50)$ \\
\hline & Total Patellar Mobility mm & $12.2(4.63)$ \\
\hline & Foot Posture index & $4.4(4.44)$ \\
\hline
\end{tabular}


Table 2 Patient-related (demographic, clinical and psychosocial) characteristics for 127 participants

\begin{tabular}{|c|c|c|}
\hline Demographic characteristics & $\begin{array}{l}\text { Mean (SD) age in years } \\
\text { Number (\%) of females } \\
\text { Mean (SD) height in } \mathrm{m} \\
\text { Mean (SD) body mass in } \mathrm{kg} \\
\text { Mean (SD) body mass index in } \mathrm{kg} / \mathrm{m}^{2}\end{array}$ & $\begin{aligned} 26 & (5.7) \\
84 & (66) \\
1.7 & (0.11) \\
73.5 & (18.3) \\
25.4 & (5.83)\end{aligned}$ \\
\hline Clinical characteristics & $\begin{array}{l}\text { Median (IQR) time since clinical onset in months* } \\
\text { Number (\%) with bilateral pain } \\
\text { Number (\%) with traumatic onset } \dagger \\
\text { Mean (SD) patellar temperature index (celsius) } \ddagger \S\end{array}$ & $\begin{array}{l}24(7-60) \\
67(52.8) \\
17(13.4) \\
4.7(3.55)\end{array}$ \\
\hline Psychosocial characteristics & $\begin{array}{l}\text { Mean (SD) Numerical Pain Rating Scale§ } \\
\text { Mean (SD) Self-completed Leeds Assessment of Neuropathic Symptoms and Signs pain scale (SLANSS)* } \\
\text { Mean (SD) Short-form McGill Pain Questionnaire } \\
\text { Continuous pain } \\
\text { Intermittent pain } \\
\text { Neuropathic pain } \\
\text { Affective descriptors } \\
\text { Number (\%) with low physical activity level-(IPAQ)ף } \\
\text { Mean (SD) Modified Functional Index Questionnaire§ } \\
\text { Mean (SD) Hopkins Symptom Checklist } \\
\text { Mean (SD) EQ-5D-5L } \\
\text { - Index value§ } \\
\text { Visual Analogue Scale (VAS) } \\
\text { Mean (SD) WHO Disability Assessment Scale I* } \\
\text { Mean (SD) Movement Specific Reinvestment Scale } \\
\text { Movement self-consciousness subscale } \\
\text { Conscious motor processing subscale }\end{array}$ & $\begin{array}{c}4.7(1.95) \\
6.5(5.84) \\
\\
3.1(1.95) \\
2.4(2.02) \\
0.8(1.15) \\
1.2(1.76) \\
19(15.0) \\
34.1(16.97) \\
1.3(0.42) \\
\\
0.7(0.17) \\
75.4(16.56) \\
19.4(7.04) \\
\\
13.3(6.69) \\
17.4(5.75)\end{array}$ \\
\hline
\end{tabular}

Figure 1 Participant flow chart.

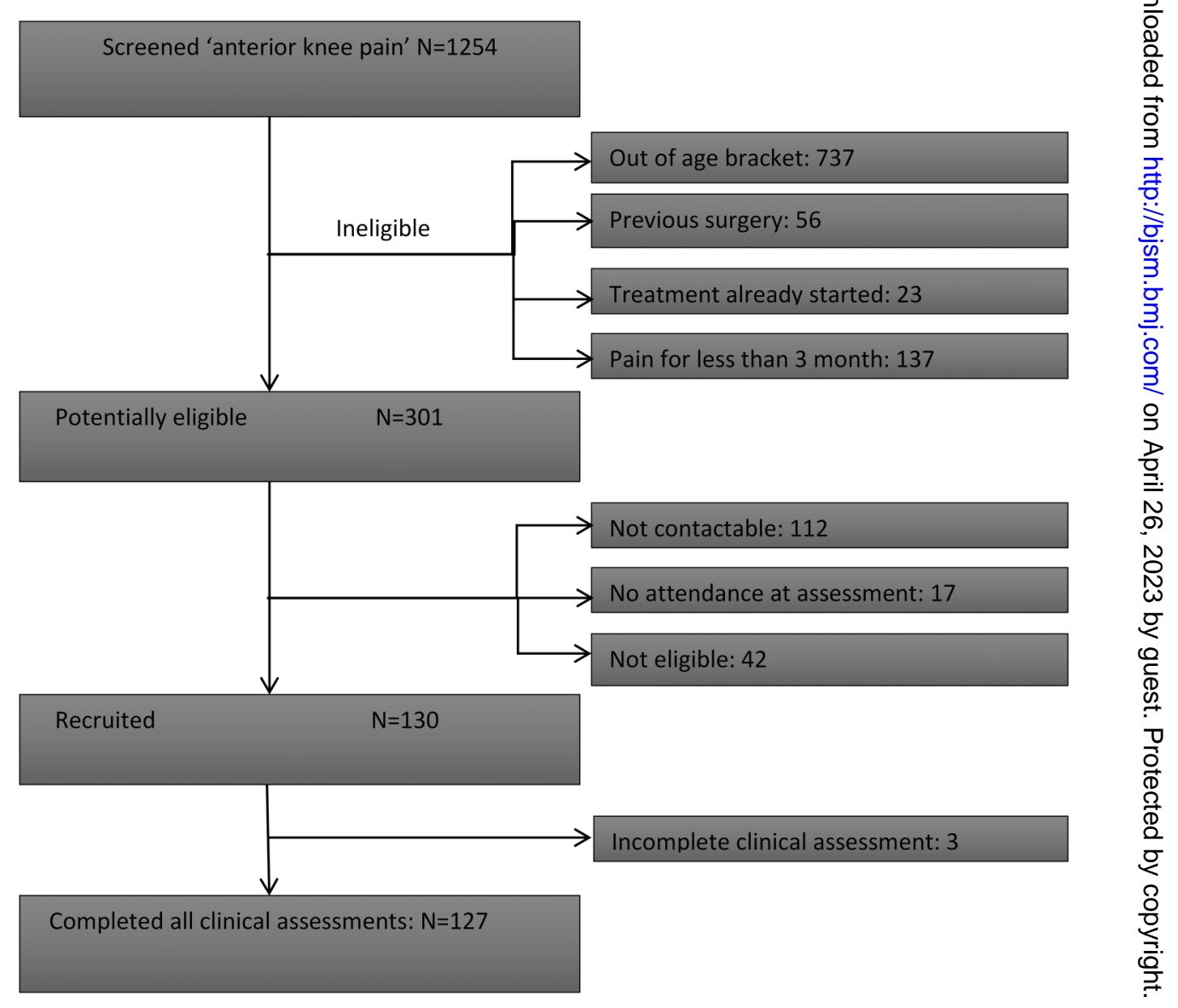


Table 3 Distribution of 127 participants into predetermined subgroups using different SD from population norms

\begin{tabular}{|c|c|c|c|c|c|}
\hline & \multirow[b]{2}{*}{$\begin{array}{l}\text { Published population norm } \\
\text { Mean (SD) }\end{array}$} & \multicolumn{4}{|c|}{ SD from published population norm } \\
\hline & & $\begin{array}{l}1 \text { SD } \\
\mathrm{n}(\%)\end{array}$ & $\begin{array}{l}1.5 \text { SD } \\
\mathrm{n}(\%)\end{array}$ & $\begin{array}{l}2 \text { SD } \\
\text { n (\%) }\end{array}$ & $\begin{array}{l}2.5 \text { SD } \\
\text { n (\%) }\end{array}$ \\
\hline Lower limb biarticular tightness & & $35(27.6)$ & $12(9.4)$ & $8(6.3)$ & $3(2.4)$ \\
\hline Quadriceps & $132.21(16.39)$ & $33(30)$ & $19(15.5)$ & $12(9.4)$ & $3(2.4)$ \\
\hline Hamstrings & $\begin{array}{l}\text { Male: } \\
142.55 \text { (6.85) } \\
\text { Female: } \\
153.66(11.13)\end{array}$ & $31(24.4)$ & $18(14.2)$ & $9(7.1)$ & $4(3.1)$ \\
\hline Gastrocnemius & $35.22(6.59)$ & $74(58.3)$ & $46(36.2)$ & $38(29.1)$ & $24(18.9)$ \\
\hline Hip abductor weakness & $\begin{array}{l}\text { Male: } \\
\text { Age <30: } 185(37.6) \\
\text { Age } \geq 30: 163(37.4) \\
\text { Female: } \\
\text { Age <30: } 114(31.8) \\
\text { Age } \geq 30: 102(26.0)\end{array}$ & $112(88.2)$ & $100(78.7)$ & $66(52)$ & $38(29.9)$ \\
\hline Quadriceps weakness & $\begin{array}{l}\text { Male: } \\
\text { Age <30: } 242(55.8) \\
\text { Age } \geq 30: 236(43.8) \\
\text { Female: } \\
\text { Age <30: } 160(26.4) \\
\text { Age } \geq 30: 157(41.9)\end{array}$ & $125(98.4)$ & $119(93.7)$ & $115(90.6)$ & $104(81.9)$ \\
\hline Patellar hypomobility & $26.2(5.8)$ & $122(96.1)$ & $111(87.4)$ & $89(70.1)$ & $63(49.6)$ \\
\hline Patellar hypermobility & $26.2(5.8)$ & 0 & 0 & 0 & 0 \\
\hline Foot pronation & $4(3)$ & $43(33.9)$ & $18(14.2)$ & $17(13.4)$ & $3(2.4)$ \\
\hline No subgroup & & 0 & $1(0.8)$ & $2(1.6)$ & $7(5.5)$ \\
\hline
\end{tabular}

score was in $\mathrm{Nm}$ because of the lack of available population data normalised for weight. Percentages of participants falling into individual subgroups and into multiple subgroups were estimated, with 95\% CIs calculated using exact binomial methods. Sensitivity analysis was undertaken using different SDs from the published population norms (1.5 SD, 2.0 SD and 2.5 SD).

Second the data were explored using two other approaches; hierarchical agglomerative cluster analysis (using SPSS) and latent profile analysis (using Latent Gold). Hierarchical agglomerative cluster analysis, a bottom-up approach to partitioning participants into subgroups based on the similarity (or distance) of the set of variables (eg, clinical tests or measures), and latent profile analysis, a statistical method of estimating the probability of individuals' membership of latent (or unknown) classes (or subgroups) based on a set of variables (eg, clinical tests or measures), in which it is assumed that the variables are independent, given the class membership. For the hierarchical agglomerative cluster analysis, Ward's method was used, Euclidean distance squared and standardised the data using the Z-scores. The number of subgroups was based on the number which could be supported within a clinical context. ${ }^{25}$

For latent profile analysis, Akaike information criterion (AIC) and Bayesian information criterion (BIC) were computed for each model to aid the choice of model and hence the number of subgroups. ${ }^{26}$ Both methods, hierarchical clustering and latent profile analysis were performed independently and parallel to each other by two separate authors of this paper. In these analyses data were used from each flexibility test separately and strength normalised for body mass $(\mathrm{Nm} / \mathrm{kg})$. The mean and SD of test scores are reported for each subgroup in each approach and analysis of variance (ANOVA) was performed to test for significant differences in individual test scores between the groups. The differences between means of other patient characteristics were also explored using ANOVA. In both sets of ANOVAs, when overall differences were statistically significant $(p<0.05)$, multiple comparisons between subgroups were performed using Tukey's B (Wholly Significant Difference) test; ${ }^{27}$ if observed subgroup variances differed substantially, the sensitivity to the equal variances assumption was assessed by also performing the Games-Howell test. ${ }^{28}$ Comparisons between subgroups for gender and activity were made using $\chi^{2}$-tests, with pairwise multiple comparisons using Bonferroni correction of $\mathrm{p}$ values when overall differences were statistically significant $(p<0.05)$.

\section{Approvals, consent and licenses}

The study received ethical approval from NRES Committee North West-Greater Manchester North, REC reference: 11/ NW/0814 and University of Central Lancashire (UCLan) Built, Sport and Health (BuSH) Ethics Committee Reference Number: BuSH 025. R\&D approval was also obtained from each participating NHS trust and licenses for the questionnaire instruments obtained, where required.

\section{RESULTS}

One hundred and thirty participants were recruited, three participants did not have a complete set of seven clinical test scores and were removed from further analyses (table 1). The study cohort was predominantly female and on average was slightly overweight, the mean age was 26 years (SD 5.7; table 2).

No participant met the à priori criteria for hypermobility, few for lower limb biarticular muscle tightness $(27.6 \%$, 95\% CI $20.0 \%$ to $36.2 \%)$ or foot pronation $(33.9 \%$, $95 \%$ CI $25.7 \%$ to $42.8 \%$ ) but most met the criteria for quadriceps weakness (98.4\%, 95\% CI $94.4 \%$ to $99.8 \%)$, patellar hypomobility $(96.1 \%, 95 \%$ CI $91.1 \%$ to $98.7 \%)$ and hip abductor weakness (88.2\%, 95\% CI $81.3 \%$ to $93.2 \%)$. Consequently, most participants $(89.8 \%, 95 \%$ CI $83.1 \%$ to $94.4 \%)$ met the criteria for at least three subgroups: $40.2 \%$ fell into three subgroups; $44.1 \%$ 




Figure 2 Subgrouping of participants based on cut-offs 1 SD from population-based mean* * Based on 125 participants with quadriceps weakness; in addition there were 2 participants without quadriceps weakness ( 1 hypomobility of patella only, 1 hypomobility of patella and foot pronation).

into four subgroups and 5.5\% into five subgroups (figure 2). Even when the most extreme values of $2.5 \mathrm{SD}$ was considered, only $38.6 \%(n=49)$ fell into just one subgroup.

Preliminary analysis of both hierarchical cluster and latent profile analysis, showed a similar mean hamstring length across subgroups, therefore this variable was excluded from the final analyses for both approaches. For the latent profile analysis approach, the AIC and BIC suggested either a three or five cluster solution as the best fit to the data. Three subgroups were chosen, as this would be more feasible to implement in practice and partitioning into five subgroups did not offer any further insight into potential treatment regimens.

Both classification methods, hierarchical clustering and latent profile analysis, generated subgroups which, on interpretation of the mean test scores across the six clinical assessments, could be given the same nomenclature (table 4): there was a 'strong' subgroup, a 'weak and tighter' subgroup, and a 'weak and pronated foot' subgroup. In both methods, the 'strong' subgroup exhibited the highest mean quadriceps and hip abductor strength with the most flexible rectus femoris and subgroup membership was highly consistent. The 'weak and tighter' subgroup, exhibited weak mean quadriceps and hip abductor strength and were less flexible (in one of the two assessments). The 'weak and pronated foot' subgroup exhibited the highest mean FPI, and in the hierarchical clustering method, this was also accompanied by the greatest patellar mobility. However, only about half of the participants were consistently classified across the hierarchical cluster and latent profile analysis methods for these two subgroups (table 5).

Table 6 shows the comparison of other patient-related factors across the three subgroups generated by each of the methods. The 'strong' subgroup had more males, lower body mass index (BMI) and higher levels of physical activity; they also exhibited lower pain scores with significantly lower SLANSS; function as measured by the MFIQ was significantly better than the 'weak and tight' subgroup when classified by the latent profile analysis, and there was a trend towards better quality of life compared to the other subgroups. The 'weak and tighter' subgroup had significantly higher BMI and worse MFIQ scores when classified by the latent profile analysis, and there was a trend towards low physical activity and longer duration of PFP, when classified by the hierarchical cluster analysis. The 'weak and pronated foot' subgroup was significantly younger at time of first assessment and had the shortest duration since the onset of their PFP according to both classification methods.

Table 4 Mean test scores across the three subgroups generated by hierarchical cluster and latent profile analysis

\begin{tabular}{|c|c|c|c|c|}
\hline Subgroup & $\begin{array}{l}\text { WEAK and TIGHTER } \\
\text { Mean (SD) }\end{array}$ & $\begin{array}{l}\text { STRONG } \\
\text { Mean (SD) }\end{array}$ & $\begin{array}{l}\text { WEAK and PRONATED FOOT } \\
\text { Mean (SD) }\end{array}$ & ANOVA \\
\hline Hierarchical clustering & $\mathrm{N}=49$ & $\mathrm{~N}=29$ & $\mathrm{~N}=49$ & \\
\hline Rectus Femoris Length ${ }^{0}$ & $121.8(19.48)$ & $140.7(17.06)^{*}$ & $130.4(19.21)$ & $F=9.26, p<0.001$ \\
\hline Gastrocnemius Length $^{0}$ & $22.3(9.71) \dagger$ & $28.0(6.51) \dagger$ & $33.1(11.21) \dagger$ & $F=14.98, p<0.001$ \\
\hline $\begin{array}{l}\text { Quadriceps Strength } \\
\mathrm{Nm} / \mathrm{kg}\end{array}$ & $0.84(0.32)$ & $1.65(0.53)^{*}$ & $0.82(0.32)$ & $F=53.01, p<0.001$ \\
\hline $\begin{array}{l}\text { Abductor Strength } \\
\mathrm{Nm} / \mathrm{kg}\end{array}$ & $0.79(0.30)$ & $1.69(0.46)^{*}$ & $0.83(0.29)$ & $F=75.11, p<0.001$ \\
\hline Total Patellar Mobility mm & $10.0(3.55)$ & $10.8(3.03)$ & $15.4(4.61)^{*}$ & $F=27.12, p<0.001$ \\
\hline Foot Posture index & $3.3(4.16)$ & $3.0(5.28)$ & $6.3(3.49)^{*}$ & $F=8.22, p<0.001$ \\
\hline Latent profile analysis & $\mathrm{N}=50$ & $\mathrm{~N}=28$ & $\mathrm{~N}=49$ & \\
\hline Rectus Femoris Length ${ }^{0}$ & $119.1(18.06)^{*}$ & $140.6(16.91)$ & $133.5(18.99)$ & $F=14.58, p<0.001$ \\
\hline Gastrocnemius Length $^{0}$ & $28.7(11.08)$ & $28.2(6.29)$ & $26.6(12.39)$ & $F=0.53, p=0.59$ \\
\hline $\begin{array}{l}\text { Quadriceps Strength } \\
\mathrm{Nm} / \mathrm{kg}\end{array}$ & $0.62(0.24) \dagger$ & $1.68(0.52) \dagger$ & $1.04(0.23) \dagger$ & $F=97.54, p<0.001$ \\
\hline $\begin{array}{l}\text { Abductor Strength } \\
\mathrm{Nm} / \mathrm{kg}\end{array}$ & $0.60(0.18) \dagger$ & $1.73(0.42) \dagger$ & $1.02(0.21) \dagger$ & $F=167.69, p<0.001$ \\
\hline Total Patellar Mobility mm & $12.5(3.77$ & $10.5(3.15)$ & $12.8(5.84)$ & $F=2.55, p=0.083$ \\
\hline Foot Posture index & $2.80(4.35)$ & $3.71(5.33)$ & $6.37(3.11)^{*}$ & $F=9.51, p<0.001$ \\
\hline
\end{tabular}


Table 5 Comparison of subgroup membership between the two classification methods

\begin{tabular}{|c|c|c|c|c|c|}
\hline \multirow{2}{*}{$\begin{array}{l}\text { Subgroups generated by } \\
\text { Hierarchical cluster analysis }\end{array}$} & & \multicolumn{3}{|c|}{ Latent profile analysis } & \multirow[t]{2}{*}{ Total } \\
\hline & & 'Weak and tight' & 'Strong' & 'Weak and pronated foot' & \\
\hline & 'Weak and tighter' & 25 & 1 & 23 & 49 \\
\hline & 'Strong' & 1 & 26 & 2 & 29 \\
\hline & 'Weak and pronated foot' & 24 & 1 & 24 & 49 \\
\hline Total & & 50 & 28 & 49 & 127 \\
\hline
\end{tabular}

\section{DISCUSSION}

The present findings suggest that three subgroups of patients with PFP may be identified using six low cost, simple clinical assessment, tests that can be applied in routine practice. This study provides an important first step in deducing whether targeted intervention for patients with PFP may be a useful strategy that ultimately leads to improved outcomes for patients. Previous work on subgrouping has mostly focused on using imaging techniques ${ }^{13-16}$ rather than on clinical testing; the small number of studies which have had a greater clinical focus have been small scale with a total of just 71 patients across two studies $^{17} 18$ these may be underpowered to detect subgroups. Although it was anticipated that separate subgroups would be identified by each of the clinical assessment tests, this was not the case. In part, this may be because of inadequately defined á priori diagnostic thresholds available in the literature, but even applying more extreme thresholds suggested most participants fell into more than one predetermined subgroup (table 3). Multiple predetermined subgroup membership was confirmed by hierarchical cluster and latent profile analysis, which generated three novel subgroups based on a combination of test scores. A 'strong' subgroup had the highest hip abductor and quadriceps strength mean scores and greatest rectus femoris length, while a 'weak and tighter' group had low mean scores for hip abductor and quadriceps strength and evidence of less flexibility, although the 'weak and pronated foot' subgroup appeared to be reliant on the results of just the FPI in the latent profile analysis, greater patellar mobility additionally appeared to be an important factor in the hierarchical cluster analysis (table 4). Using different populations to that reported in this paper previous researchers ${ }^{17} 18$ have proposed four rather than three clinical subgroups of patients with PFP. However, in common with the results reported here both previous papers describe a tight or hypomobile group that included

Table 6 Patient-related factors distributed across the three subgroups generated by the hierarchical cluster and latent profile analysis

\begin{tabular}{|c|c|c|c|c|}
\hline Subgroup & $\begin{array}{l}\text { WEAK and TIGHTER } \\
\text { Mean (SD) }\end{array}$ & $\begin{array}{l}\text { STRONG } \\
\text { Mean (SD) }\end{array}$ & $\begin{array}{l}\text { WEAK and PRONATED FOOT } \\
\text { Mean (SD) }\end{array}$ & $\begin{array}{l}\text { Test statistic } \\
\text { and } p \text { value }\end{array}$ \\
\hline Hierarchical cluster analysis & $\mathrm{N}=49$ & $\mathrm{~N}=29$ & $\mathrm{~N}=49$ & \\
\hline Age & $26.9(5.34)$ & $28.3(6.15)^{*}$ & $24.9(5.36)^{*}$ & $F=3.85, p=0.024$ \\
\hline Gender (\% male) & $13(26.5 \%)$ & $17(53.6 \%) \dagger$ & $13(26.5 \%)$ & $\chi^{2}=10.29, p=0.006$ \\
\hline BMI & $25.8(5.37)$ & $23.5(4.44)$ & $26.2(6.77)$ & $\mathrm{F}=2.12, \mathrm{p}=0.12$ \\
\hline Physical activity level (low) & $10(20.8 \%)$ & $2(6.9 \%)$ & $7(15.2 \%)$ & $\chi^{2}=2.69, p=0.26$ \\
\hline Movement specific reinvestment scale & $31.4(11.33)$ & $29.0(12.50)$ & $30.9(10.66)$ & $F=0.418, p=0.66$ \\
\hline Time from onset & $58.0(63.77)$ & $45.2(57.5)$ & $34.1(43.15)$ & $F=2.22, p=0.11$ \\
\hline HSCL (depression) & $1.3(0.38)$ & $1.3(0.57)$ & $1.3(0.36)$ & $F=0.066, p=0.94$ \\
\hline Pain (NPRS) & $5.2(1.88)$ & $4.1(1.68)$ & $4.5(2.08)$ & $F=2.84, p=0.062$ \\
\hline SLANSS (total) & $8.1(5.85)^{*}$ & $4.4(5.58)^{*}$ & $6.1(5.62)$ & $F=3.98, p=0.021$ \\
\hline EQ-5D-5 L VAS & $75.9(14.40)$ & $77.9(17.23)$ & $73.5(18.22)$ & $F=0.67, p=0.52$ \\
\hline WHO Disability Assessment Scale II & $20.9(7.27)$ & $17.5(8.27)$ & $19.2(5.70)$ & $F=2.24, p=0.11$ \\
\hline Modified Functional Index Questionnaire & $37.0(16.99)$ & $28.0(15.45)$ & $35.0(17.21)$ & $F=2.72, p=0.070$ \\
\hline Latent profile analysis & $\mathrm{N}=50$ & $\mathrm{~N}=28$ & $\mathrm{~N}=49$ & \\
\hline Age & $27.1(5.56)$ & $28.1(5.99)^{*}$ & $24.8(5.26)^{*}$ & $F=3.74, p=0.026$ \\
\hline Gender (\% male) & $8(16.0 \%)^{*}$ & $17(60.7 \%)^{*}$ & $18(36.7 \%)$ & $\chi^{2}=16.32, p<0.001$ \\
\hline BMI & $28.3(6.81) \dagger$ & $23.2(4.60)$ & $23.7(3.89)$ & $F=12.24, p<0.001$ \\
\hline Physical activity level (low) & $8(16.7 \%)$ & $2(7.1 \%)$ & $9(19.1 \%)$ & $\chi^{2}=2.03, p=0.36$ \\
\hline Movement specific reinvestment scale & $30.6(10.89)$ & $28.6(12.34)$ & $31.9(11.18)$ & $\mathrm{F}=0.77, \mathrm{p}=0.47$ \\
\hline Time from onset & $46.7(54.67)$ & $47.2(57.70)$ & $44.5(56.93)$ & $F=0.03, p=0.97$ \\
\hline HSCL (depression) & $1.3(0.37)$ & $1.23(0.45)$ & $1.3(0.46)$ & $\mathrm{F}=0.21, \mathrm{p}=0.81$ \\
\hline Pain (NPRS) & $5.0(2.03)$ & $4.14(1.76)$ & $4.6(1.93)$ & $F=1.96, p=0.15$ \\
\hline SLANSS (total) & $6.5(5.36)$ & $4.14(5.62)^{*}$ & $7.8(6.11)^{*}$ & $F=3.56, p=0.031$ \\
\hline EQ-5D-5 L VAS & $72.3(18.54)$ & $80.4(15.61)$ & $75.8(14.40)$ & $\mathrm{F}=2.18, \mathrm{p}=0.12$ \\
\hline WHO Disability Assessment Scale II & $20.4(6.59)$ & $16.8(8.05)$ & $20.0(6.60)$ & $F=2.59, p=0.079$ \\
\hline Modified Functional Index Questionnaire & $38.1(16.98)^{*}$ & $28.3(16.52)^{*}$ & $33.5(16.46)$ & $\mathrm{F}=3.18, \mathrm{p}=0.045$ \\
\hline
\end{tabular}

*Subgroup pairs different $(p<0.05)$.

tDifferent from each of the other two subgroups $(p<0.05)$.

BMI, body mass index; HSCL, Hopkins Symptom Checklist; NPRS, Numerical Pain Rating Scale; SLANSS, Self-completed Leeds Assessment of Neuropathic Symptoms and Signs pain scale; VAS, visual analogue scale. 
measurements of rectus femoris and gastrocnemius length. Both previous papers also describe a weak group where weakness in the quadriceps and hip muscles were identified by a combination of visual inspection and functional testing rather than through specific objective testing using dynamometry. It is interesting to note that three independent studies performed in different countries USA, ${ }^{17}$ Australia ${ }^{18}$ and the UK, each with a slightly different PFP population and each using slightly different methods have reported some consistency in subgroups of patients with PFP.

Therapist fidelity to the assessment process was high with only three patients with incomplete clinical assessments. This suggests that the assessments were feasible in practice within both primary and tertiary care physiotherapy clinics. Exploratory analyses also suggested that clinical assessment test scores of hamstring length are not informative in terms of subgrouping. From a clinical perspective these results are very interesting as hamstring stretching is often a component of physiotherapy treatment regimens for PFP. While hamstring tightness does not appear to be an important factor for subgrouping in PFP, our results compared to normative data found tight hamstrings in $24.4 \%(\mathrm{n}=31)$ participants indicating that some patients may benefit from treatment. The research therapists conducting the tests found the assessment of quadriceps strength easier than the hip abductor measurement and we test scores were moderately highly correlated $(r=0.72)$, so further investigation of the 'added value' of performing both tests is merited. Further work to identify the optimal thresholds for individual and combined clinical assessment tests which best classify PFP participants into the three novel subgroups is currently being undertaken. This work could potentially reduce the burden of assessment by reducing the number of tests required.

Other measures were included to assess patient characteristics such as the Hopkins Symptom Checklist and the Movement Specific Reinvestment Scale. However, these tests did not seem to contribute significantly to our understanding of subgroups or were difficult to administer, for example, the Short-form McGill Pain Questionnaire, so we propose to exclude these tests in future studies of subgrouping patients with PFP. WHODAS II scores were moderately highly correlated (Spearman's $r=-0.68$ ) with the EQ-5D-5 L, which has become firmly established as the 'gold standard' quality-of-life outcome measure for musculoskeletal physiotherapy practice in the $\mathrm{UK}^{29}$ so on this basis we would also exclude the WHODAS II from further studies.

The baseline characteristics of the participants suggest that the study population was representative of patients with PFP attending physiotherapy clinics. ${ }^{23} 3031$ The ratio of females to males was 2 to 1 , a high proportion had bilateral pain (53\%), and only a small percentage (13.8\%) of patients reported a traumatic onset of pain. While the BMI profile of this cohort might be higher than expected for athletes with PFP, it was still lower than that of the UK general population and reflects that this was a general clinical population. ${ }^{32}$ Mean clinical assessment test scores were also consistent with published findings for PFP patients. ${ }^{33-35}$ Across the whole sample, pain scores were relatively low, and function scores, levels of physical activity and quality-of-life scores were relatively good, as might be expected for what is considered a relatively low grade bothersome musculoskeletal condition. There were marked differences in the relative frequency of men and women across the subgroups. Although overall there were about twice as many women as men in the study population, there were relatively more men in the 'strong' group. While this observation might be considered inevitable because females tend to have lower muscle strength than males, about half or 4 in 10 were women in this subgroup, dependent on the method used (table 6). Analysis suggested that subgroups were stable for female participants but the number of males were too small for further analysis (data not shown). Further research should focus on potential differences in characteristics between subgroups and on investigating whether there are differences in subgroups between genders.

There were also differences between the subgroups with respect to some of the other participant characteristics. While it is not possible from this cross-sectional study to identify the direction of the relationship between the test scores and these other characteristics, they may provide further insights into aetiology or sequelae, which could guide further research on preventative strategies or therapeutic management. The 'weak and tighter' subgroup, generated by latent class analysis, had significantly higher mean BMI, with the majority being overweight and lowest physical activity, when subgroups were generated by the hierarchical approach. Being overweight has been associated with patellar cartilage loss. ${ }^{36}{ }^{37}$ The speculated relationship between patellofemoral pain and patellofemoral osteoarthritis and the known relationship between obesity and knee osteoarthritis suggests that this observation is worthy of further investigation. ${ }^{6}$ Whether the development of patellofemoral OA is potentially greater in this group compared to other two groups is at this stage highly speculative. In the short term it might however, point towards the need for adjunct strategies to promote activity and encourage weight loss in this subgroup, in addition to strengthening and flexibility exercises. While lower limb muscle weakness in patients with PFP is well known, it was more surprising that a 'strong' subgroup existed with a trend towards less pain, higher function and better quality of life. This might suggest that the other well-known observation in patients with PFP, that of poor neuromuscular control, is important and interventions focusing on movement control are required. ${ }^{38} 39$ The significantly younger age of the 'weak and pronated foot' group is interesting but initial suggestions of a developmental issue, are tempered by us specifically recruiting over 18-year-olds to minimise the chance of 'growth spurt' problems. Other studies have demonstrated higher levels of passive ankle dorsiflexion in adolescents with knee pain ${ }^{40}$ and this might suggest strategies including foot orthoses are warranted specifically for this subgroup.

\section{Limitations}

This was not an efficacy trial and there are no outcome data following treatment. Therefore, it is unclear whether using the three subgroups suggested by this study will have any impact on modifying clinical practice or more importantly on improving patient outcomes. We considered that we needed at least 150 participants but recruited 130 of which 127 had sufficient data to be included in the exploratory analyses. Recruitment had to close because of time constraints. Although the target sample size was not reached, CIs for subgroups based on a priori thresholds are relatively precise and similar subgroups across hierarchical cluster and latent profile analysis have been generated. However, given the small number of men in the sample, we could not confirm that subgroupings are similar in different genders. Additionally the study focused specifically on the young adult population aged $18-40$ years, so it is unknown if these subgroups are relevant to adolescents or older patients.

There are a myriad of different approaches for subgrouping data and these will tend to give different results for the same data set. ${ }^{25}$ We chose to explore the data using two different 
approaches to provide some internal validation. We were to some extent reassured that generated subgroups could be given the same nomenclature. However, there were important differences in participant characteristics and the mean test scores between the groups. This makes clinical interpretation difficult. The two approaches differ in how they generate subgroups with latent profile analysis splitting the sample into smaller groups whereas hierarchical agglomerative clustering has a bottom-up approach. Also, latent profile analysis differs from cluster analysis methods in that individuals are not assigned definitively to classes based on a chosen distance measure but are typically assigned to classes based on probabilities of membership of each class, usually estimated via maximum-likelihood estimation of the parameters of a specified model. Unlike cluster analysis, there is no requirement to explicitly scale each variable as the classification is based directly on the distributional properties of the variables and classifications are therefore unaffected by the choice of a variable's scale. Owing to these features, latent profile analysis is increasingly considered a better analytical approach to hierarchical clustering methods. ${ }^{40}$ It also provides information on the most likely number of clusters (by using the AIC and BIC), whereas this is more difficult to assess in hierarchical clustering methods. However, hierarchical clustering may more closely reflect clinical decision-making where test scores are assessed sequentially to build up a picture of the main problem of the patient. Further validation of the subgroups using other data sets is required which would also provide further information on the relevance of patellar mobility and other patient characteristics. Furthermore, it will be important to determine if optimising treatments based on subgroups will improve patient outcomes.

\section{SUMMARY AND CONCLUSIONS}

Three subgroups of patients with PFP have emerged based on six clinical assessment tests. A 'strong' subgroup had the greatest rectus femoris length, lowest pain scores, significantly more males, better function and better quality-of-life and were the oldest. A 'weak and tighter' subgroup had significantly higher BMI, MFIQ and SLANSS with a trend towards lower physical activity levels and the longest duration of PFP. A 'weak and pronated foot' subgroup had the greatest patellar mobility, was significantly younger at time of first assessment and had the shortest duration of PFP. The study suggests that the six assessment procedures are feasible for therapists in primary care and hospital settings to perform in routine practice. We propose to undertake further work to validate these subgroups using external data sets, to examine optimal thresholds to assign participants to groups and, to assess whether more targeted intervention, based on these subgroups, would improve patient compliance and outcome, and as a result be more cost-effective.

\section{What are the findings?}

- Three subgroups of patellofemoral patients have been identified.

The subgroups are: 'strong'; 'weak and tighter'; 'weak and pronated'.

- Six simple low cost clinical tests can be used to identify the subgroups.

\section{How might it impact on clinical practice in the future?}

Targeted intervention based on these subgroups may improve patient outcomes.

Acknowledgements The authors would like to thank all the patients who kindly volunteered to take part in this study. This work was supported by Arthritis Research UK grant number 19950 and involves collaboration with the Arthritis Research UK Centre for Sport, Exercise and Osteoarthritis. Arthritis Research UK Musculoskeletal Pain CSG, also funded a Think Tank meeting where our research group consisting of academics with expertise in patellofemoral pain, biomechanists, psychosocial aspects related to injury rehabilitation adherence, experts in neuromuscular function, patient representative and practising physiotherapists started to review the literature to identify clinical groups. This Think Tank meeting also allowed us to develop plans for studies investigating the subgrouping and targeted intervention approach. The authors thank the following physiotherapists for performing the research assessments Steve Hill, Stephen Kirk, Gary McCall, Christine Dewsbury, Kim Patterson and Sophie Chatwin. The authors would also like to thank the following service mangers for their support Keith Mills, Elaine Nicholls, Barbara Sharp, Chante Ostler and Kim Patterson. The authors would also like to thank Professors Remco Polman and Rich Masters and to David Turner, for support and advice during the early stages of project development. The authors would like to thank Brian Francis for his advice on latent profile analysis and its application. The TIPPs team acknowledge the support of the National Institute for Health Research, through the Comprehensive Clinical Research Network.

Contributors JS contributed to study conception, design and attained project funding, project management and manuscript preparation. JJ contributed to project management, interpretation of data and manuscript preparation. MC and JR contributed to study conception, design and attained project funding. JR also contributed to project management and manuscript preparation. EW contributed to study conception, design and manuscript revising. CS contributed to study conception, design and analysis and interpretation of data. CS also contributed to project management and manuscript preparation. MS contributed to study design and attained project funding. MS also contributed to project management interpretation of data and manuscript preparation. DM contributed to study conception, design and analysis and interpretation of data. DM also contributed to project management and manuscript preparation. JD contributed to study conception design and attained project funding. JD also contributed to drafting the manuscript. $\mathrm{RH}$ contributed to service user patient involvement throughout the project. VB contributed to the study design, data collection, protocol and quality assurance of strength measurements and interpretation and drafting of the manuscript. ER contributed to the study design and facilitated the acquisition of data. NA contributed to study conception, design and manuscript preparation. PD contributed to study conception, design, project management, data analysis and interpretation and manuscript preparation. All authors read and approved the final manuscript.

Competing interests None declared.

Ethics approval NRES Committee North West-Greater Manchester North.

Provenance and peer review Not commissioned; externally peer reviewed.

Open Access This is an Open Access article distributed in accordance with the Creative Commons Attribution Non Commercial (CC BY-NC 4.0) license, which permits others to distribute, remix, adapt, build upon this work non-commercially, and license their derivative works on different terms, provided the original work is properly cited and the use is non-commercial. See: http://creativecommons.org/ licenses/by-nc/4.0/

\section{REFERENCES}

1 Domenech J, Sanchis-Alfonso V, López L, et al. Influence of kinesiophobia and catastrophizing on pain and disability in anterior knee pain patients. Knee Surg Sports Traumatol Arthrosc 2013;21:1562-8.

2 Stathopulu E, Baildam E. Anterior knee pain: a long-term follow-up. Rheumatology 2003;42:380-2

3 Price AJ, Jones J, Allum R. Chronic traumatic anterior knee pain. Injury 2000;31:373-8

4 Sandow MJ, Goodfellow JW. The natural history of anterior knee pain in adolescents. J Bone Jt Surg (Br) 1985;67:36-8.

5 Thomas M, Wood L, Selfe J, et al. Anterior knee pain in younger adults as a precursor to subsequent patellofemoral osteoarthritis: a systematic review. BMC Musculoskelet Disord 2010;11:201.

6 Crossley KM. Is patellofemoral osteoarthritis a common sequela of patellofemoral pain? Br J Sports Med 2014;48:409-10. 
7 Rankin G, Rushton A, Oliver P, et al. Chartered Society of Physiotherapy's identification of national research priorities for physiotherapy using a modified Delphi technique. Physiotherapy 2012;98:260-72.

8 Witvrouw E, Callaghan MJ, Stefanik JJ, et al. Patellofemoral pain: consensus statement from the 3rd International Patellofemoral Pain Research Retreat held in Vancouver, September 2013. Br J Sports Med 2014;48:411-14.

9 Davis I, Powers C. Patellofemoral pain syndrome: proximal, distal, and local factors, an international retreat, April 30-May 2, 2009, Fells Point, Baltimore, MD. J Orthop Sports Phys Ther 2010;40:A1-16.

10 Powers C, Bolgla L, Callaghan M, et al. 2nd International Patellofemoral Research Retreat, Ghent, Belgium, patellofemoral pain: proximal, distal, and local factors. J Orthop Sports Phys Ther 2012;42:A1-54.

11 Brennan GP, Fritz JM, Hunter SJ, et al. Identifying subgroups of patients with acute/ subacute "nonspecific" low back pain: results of a randomized clinical trial. Spine 2006;31:623-31.

12 Hill J, Dunn K, Lewis M, et al. A primary care back pain screening tool: identifying patient subgroups for initial treatment. Arthritis Rheum 2008;59:632-41.

13 Naslund J, Naslund UB, Odenbring S, et al. Comparison of symptoms and clinical findings in subgroups of individuals with patellofemoral pain. Physiother Theory Pract 2006;22:105-18.

14 Sheehan F, Derasari A, Fine K, et al. Q-angle and J-sign: indicative of maltracking in subgroups in patellofemoral pain. Clin Orthop Relat Res 2010;468:266-75.

15 Harbaugh C, Wilson N, Sheehan F. Correlating femoral shape with patellar kinematic in patients with patellofemoral pain. J Orthop Res 2010;28:865-72.

16 Dierks T, Manal K, Hamill J, et al. Lower extremity kinematics in runners with patellofemoral pain during a prolonged run. Med Sci Sports Exerc 2011:43:693-700.

17 Selhorst M, Rice W, Degenhart T, et al. Evaluation of a treatment algorithm for patients with patellofemoral pain syndrome: a pilot study. Int I Sports Phys Ther 2015;10:178-88.

18 Keays S, Mason M, Newcombe P. Individualised physiotherapy in the treatment of patellofemoral pain. Physiother Res Int 2015;20:22-36.

19 Boling M, Padua D, Marshall S, et al. Gender differences in the incidence and prevalence of patellofemoral pain syndrome. Scand J Med Sci Sports 2010;20:725-30.

20 Dey M, Callaghan M, Erande R, et al. Cohort studies in patellofemoral pain syndrome the search for modifiable risk factors: a systematic review and critique abstract. J Orthop Sports Phys Ther 2012;42:A51.

21 Rathleff MS, Roos EM, Olesen JL, et al. Lower mechanical pressure pain thresholds in female adolescents with patellofemoral pain syndrome. J Orthop Sports Phys Ther 2013;43:414-21.

22 Selfe J, Callaghan M, Witvrouw E, et al. Targeted interventions for patellofemoral pain syndrome (TIPPS): classification of clinical subgroups. BMJ Open 2013;3: e003795.
23 Syme G, Rowe P, Martin D, et al. Disability in patients with chronic patellofemoral pain syndrome: a randomised controlled trial of VMO selective training versus general quadriceps training. Man Ther 2009;14:252-63.

24 Cook C, Hegedus E, Hawkins R, et al. Diagnostic accuracy and association to disability of clinical test findings associated with patellofemoral pain syndrome. Physiother Can 2010;62:17-24.

25 Everitt BS, Landau S, Leese $M$, et al. Cluster analysis (Wiley series in probability and statistics). 5th edn. John Wiley and Sons, 2011. ISBN 13: 978-04070749913.

26 Hagenaars JA, McCutcheon AL. Applied latent class analysis. Cambridge, NY: Cambridge University Press, 2002.

27 Hsu J. Multiple comparisons: theory and methods. London: Chapman \& Hall, 1996.

28 Games PA, Howell JF. Pairwise multiple comparison procedures with unequal N's and /or variances: a Monte Carlo study. J Educ Stat 1976;1:113-25.

29 Chartered Society of Physiotherapy. Measuring change in musculoskeletal physiotherapy outpatient services. http://www.csp.org.uk/documents/ eq-5d-5l-measuring-change-musculoskeletal-physiotherapy-outpatient-services (accessed Mar 2015).

30 Brown J. Physiotherapists knowledge of patellofemoral pain syndrome. $\mathrm{Br}$ J Ther Rehabil 2000;7:346-53.

31 Selfe J, Harper L, Pedersen I, et al. Four outcome measures for patellofemoral joint problems: part 1 development and validity. Physiotherapy 2001;87:507-15

32 Craig R, Mindell J, eds. Health Survey for England 2013: health, social care and lifestyles. Volume 1: Health, social care and lifestyles. Volume 2: Methods and documentation. Leeds: Health and Social Care Information Centre, 2014.

33 Skalley TC, Terry GC, Teitge RA. The quantitative measurement of normal passive medial and lateral patellar motion limits. Am J Sports Med 1993;21:728-32.

34 Witwrouw E, Lysens R, Bellemans J, et al. Intrinsic risk factors for the development of anterior knee pain in an athletic population: a two year prospective study. Am J Sports Med 2000;28:480-9.

35 Toonstra J, Mattacola CG. Test-retest reliability and validity of isometric knee-flexion and -extension measurement using 3 methods of assessing muscle strength. J Sport Rehabil 2013;Technical Notes (7).pii: 2012-0017.

36 Cicuttini F, Wluka A, Wang Y, et al. The determinants of change in patella cartilage volume in osteoarthritic knees. J Rheumatol 2002;29:2615-19.

37 Teichtahl AJ, Wluka AE, Wang $Y$, et al. Obesity and adiposity are associated with the rate of patella cartilage volume loss over 2 years in adults without knee osteoarthritis. Ann Rheum Dis 2009;68:909-13.

38 Noehren B, Scholz J, Davis I. The effect of real-time gait retraining on hip kinematics, pain and function in subjects with patellofemoral pain syndrome. $\mathrm{Br} \mathrm{J}$ Sports Med 2011;45:691-6.

39 Graci V, Salsich GB. Trunk and lower extremity segment kinematics and their relationship to pain following movement instruction during a single-leg squat in females with dynamic knee valgus and patellofemoral pain. J Sci Med Sport 2015;18:343-7.

40 Jung T, Wickrama KA. An introduction to latent class growth analysis and growth mixture modelling. Soc Personal Psychol Compass 2008;2:302-17. 